\title{
Ozone sterilization: Renewed potential for combating bacterial infections in severe burns, decubitus, and leg ulcers
}

\author{
Yasuhiro Horiuchi MD (1) \\ Division of Dermatology, Tsuruse Orthopedic Clinic, Saitama, Japan
}

To the Editor-Although we have developed powerful antibiotics, we are still faced with the dilemma of controlling bacterial infection in the skin in cases such as in burns ${ }^{1}$ or decubitus. ${ }^{2}$ The sterilizing effect of ozone, ${ }^{3}$ which has been known for a long time, involves killing bacteria by rupturing the cell wall through its action on certain cell-wall components. Thus, we may consider using ozone sterilization to treat infections in the skin that are difficult to control with antibiotics. Recently, ozone sterilization has made great progress with the invention of ozone nanobubble (ONB) water ${ }^{3}$ (NAGA, Japan), in which nanosized ozone gas particles are dissolved in water. ONB water has attracted attention as a new and safer antiseptic agent. The sterilizing effects of ozone have been reevaluated in recent years, ${ }^{4}$ and the improved form of ozone, ONB water ${ }^{3}$ could change the current perception of ozone use in the medical field. These studies ${ }^{3,4}$ indicate that ozone sterilization could potentially be used against bacteria in medical care.

In severely burned patients, ${ }^{1}$ bacterial infections on the wound can inhibit epithelialization of the wound, which sometimes leads to a prolonged battle against bacterial infections in the intensive care unit. Silver-containing compounds (silver sulfadiazine, Ag-SD) ${ }^{1}$ are being used as a viable treatment option for infections encountered in severe burns; however, the compound has serious harmful effects. It delays the wound-healing process by conferring serious cytotoxicity to the host tissues. Repeatedly cleaning the wound with physiological saline $e^{5}$ has limits in controlling bacterial growth. The overlap of these adverse conditions owing to secondary infections and/or a delay in epithelialization worsens the patient's condition. Bacterial infections of the wound are a threat to the success of the skin graft, even if it can reach artificial skin preparation and/or the skin graft. ${ }^{1}$ Thus, the sterilizing action of ozone, wherein bacteria are killed by rupturing the cell, is 30 times more effective than chlorine disinfection. ${ }^{3}$ Ozone water will greatly change the environment of burns, and will reduce the risk of infection in burns. Additionally, ozone sterilization does not lead to resistant strains and is safer because it does not damage the mucosal tissue. ${ }^{3}$ Moreover, the ozonization of saline is needed, but the utility of ONB saline has not yet been proven and ONB water has not yet been approved for medical use. Furthermore, because dissolved nanosized ozone gas particles can be maintained for 3-6 months in $\mathrm{ONB}$ water, ${ }^{3}$ it can easily be stocked at medical site.

Decubitus or pressure ulcers, ${ }^{2}$ a worldwide healthcare concern, affect patients who are bedridden for longer periods, particularly elderly individuals, due to the mechanical pressure exerted by patient's own body. These ulcers sometimes harbor secondary bacterial infections, sometimes caused by Pseudomonas

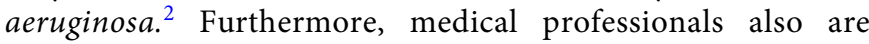

Author for correspondence: Yasuhiro Horiuchi, E-mail: tshoriuchi15@gmail.com

Cite this article: Horiuchi Y. (2020). Ozone sterilization: Renewed potential for combating bacterial infections in severe burns, decubitus, and leg ulcers. Infection Control \& Hospital Epidemiology, 41: 1247-1248, https://doi.org/10.1017/ice.2020.157 forced to continue treatments, such as cleaning and ulcer repair daily. Severe conditions that arise because of secondary bacterial infections cannot be treated successfully with saline alone. ${ }^{5}$ Thus, wound cleaning with ozone water ${ }^{6}$ can help to avoid secondary infections and can thus improve these difficult-to-treat pressure ulcers.

In countries such as Europe and the United States, where obesity is a major problem, venous ${ }^{7}$ or diabetic skin ulcers, ${ }^{8}$ are persistent, and it is difficult to control their secondary bacterial infections, such as Pseudomonas aeruginosa infection. ${ }^{9}$ In patients with leg ulcers, owing to the difficulty in treating and curing the underlying diseases such as venous varicose disorder, skin ulcers may also persist for longer periods. Similar to treating secondary infections in decubitus ulcers, cleaning and sterilization of the wound with ozone water ${ }^{6}$ will greatly contribute to and assist in maintaining and improving these intractable skin ulcers. Additionally, in these skin conditions in which daily washing is performed continuously as a part of the standardized protocol, ${ }^{5}$ it would be important to use ozone water ${ }^{3,6}$ to kill bacteria mechanically and to prevent the development of resistant bacterial strains.

Despite developing antibiotics, controlling skin bacteria infections remains an unresolved issue. Furthermore, the spread of resistant bacteria due to the widespread use of antibiotics has also become a serious worldwide medical concern, and the number of resistant strains is increasing. ${ }^{10}$ Therefore, owing to the characteristics and safety of ozone, we expect it to be used as a new countermeasure against bacterial infections in the skin. However, it is necessary to soak the wound for 30 seconds or more for the ozone water to effectively sterilize the area. Furthermore, the unlimited possibility of ozone as a antibacterial agent in various disciplines is expanding. Currently, research in the medical field and is focused more on developing cutting-edge treatments, such as developing new antibiotics for resistant bacteria; however, it is necessary to return to the root cause of the issues and to consider treatment. Even if ONB water is unavailable, ozonated water can be prepared with an ozone gas generator.

Acknowledgments. I would like to thank Editage for English language editing.

Financial support. No financial support was provided relevant to this article.

Conflicts of interest. The author reports no conflicts of interest relevant to this article.

\section{References}

1. Coban YK. Infection control in severely burned patients. World J Crit Care Med 2012;1:94-101.

2. Livesley NJ, Chow AW. Infected pressure ulcers in elderly individuals. Clin Infect Dis 2002;35:1390-1396.

3. Hayakumo S, Arakawa S, Takahashi M, Kondo K, Mano Y, Izumi Y. Effects of ozone nano-bubble water on periodontopathic bacteria and oral cells-in vitro studies. Sci Tech Adv Mater 2014;15:055003. 
4. Fitzpatrick E, Holland OJ, Vanderlelie JJ. Ozone therapy for the treatment of chronic wounds: a systematic review. Int Wound J 2018;15: 633-644.

5. Huang CY, Choong MY. Comparison of wounds' infection rate between tap water and normal saline cleansing: a meta-analysis of randomised control trials. Int Wound J 2019;16:300-301.

6. Al-Saadi H, Potapova I, Rochford ET, Moriarty TF, Messmer P. Ozonated saline shows activity against planktonic and biofilm growing Staphylococcus aureus in vitro: a potential irrigant for infected wounds. Int Wound J 2016; 13:936-942.
7. Mekkes JR, Loots MAM, Van Der Wal AC, Bos JD. Causes, investigation and treatment of leg ulcer. Brit J Dermatol 2003;148:388-401.

8. Casqueiro J, Casqueiro J, Alves C. Infections in patients with diabetes mellitus: a review of pathogenesis. Indian J Endocr Metab 2012;16: s27-s36.

9. Georgesc M, Gheorghe I, Iordache C. Virulence and resistance features of Pseudomonas aeruginosa strains isolated from chronic leg ulcers. BMC Infect Dis 2016;16:92.

10. Laxminarayan R, Duse A, Wattal C, et al. Antibiotic resistance- the need for global solutions. Lancet Infect Dis 2013;13:1057-1098. 